\title{
DESCRIPTION D'UN NOUVEAU GENRE DE BENTHIMERMITHIDAE (NEMATODA) PRÉSENTANT DES UTÉRUS MUNIS DE GLANDES ANNEXES
}

\author{
A. J. PETTER*
}

RÉSUMÉ. Description de femelles juvéniles appartenant à un nouveau genre et une nouvelle espèce de la famille des Benthimermithidae, Adenodelphis eurythenes n. g., n. sp., parasites de la cavité générale de l'Amphipode Eurythenes gryllus dans les sédiments abyssaux de la Mer de Norvège. Le genre est caractérisé principalement par la présence de glandes annexes échelonnées le long du tiers proximal des utérus.

\section{Description of a new genus of Benthimermithidae (Nematoda) with unusual uterine glands.}

SUMMARY. Juvenile females belonging to a new genus and new species of Benthimermithidae, Adenodelphis eurythenes n. gen., n. sp., parasites of the amphipod Eurythenes gryllus in abyssal sediments from the Norway Sea are described. The genus is characterized mainly by the presence of uterine glands scattered along the proximal third of the uterus.

Certains Amphipodes de l'espèce Eurythenes gryllus récoltés au cours de la campagne Norbi $^{1}$ (mer de Norvège) étaient parasités par des Nématodes qui nous ont été confiés pour étude par le CENTOB ( fig. 1).

Ces Nématodes sont repliés dans la cavité générale de l'Amphipode, entourant le tube digestif ; la plupart des Amphipodes ne sont parasités que par un seul Nématode, mais certains en possèdent plusieurs et l'un d'entre eux en contenait 8.

Il s'agit uniquement de femelles juvéniles appartenant à la famille des Benthimermithidae, mais qui présentent quelques structures tout à fait particulières que nous décrivons ici.

* Laboratoire des Vers, associé au C.N.R.S., Muséum National d'Histoire Naturelle, 61, rue de Buffon, F 75231 Paris Cedex 05.

I. La Campagne Norbi (chef de Mission Lucien Laubier) a été organisée par le COB (Centre Océanologique de Bretagne); le tri a été effectué par le CENTOB (Centre de Tri d'Océanographie biologique).

Accepté le 27 septembre 1982. 


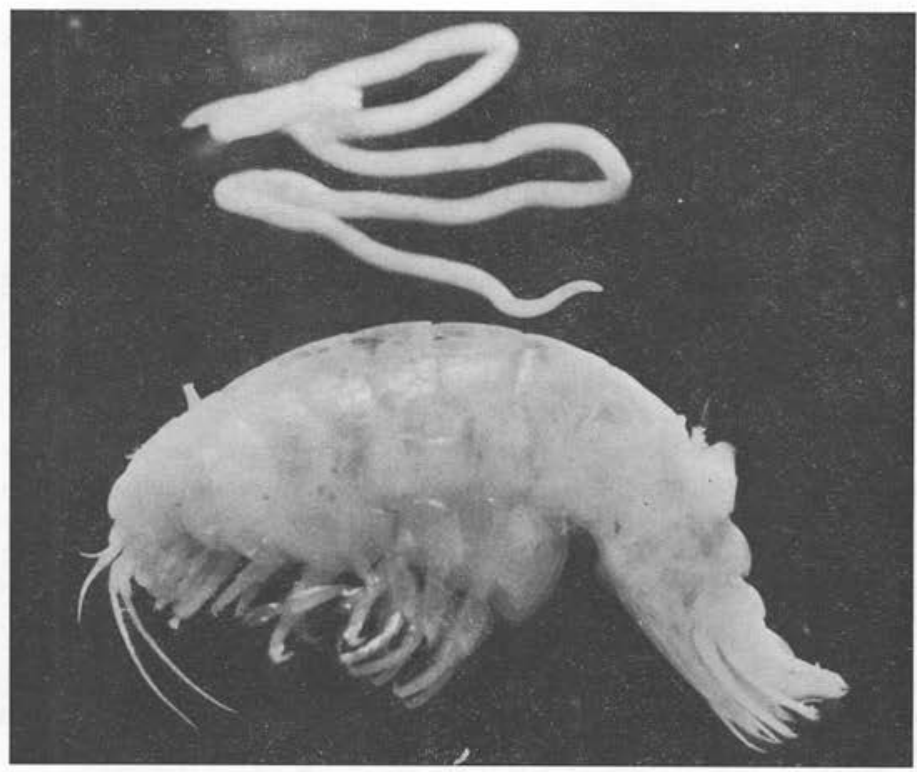

FiG. I. - Eurythenes gryllus et femelle juvénile d'Adenodelphis eurythenes extraite de sa cavité générale. $(\times 2,5)$.

\section{Adenodelphis eurythenes n. g., n. sp.}

\section{DESCRIPTION}

Matériel étudié : 39 q juvéniles trouvées dans la cavité générale de 24 Eurythenes gryllus provenant de 3 stations de la campagne Norbi : 22.07.1975.

— station no 3 , prélèvement no $17: 66^{\circ} 46^{\prime}, 8 \mathrm{~N}-01^{\circ} 35^{\prime}$, OW ; $3699 \mathrm{~m}$;

- station no 2, prélèvement no $12: 65^{\circ} 16^{\prime}, 5 \mathrm{~N}-00^{\circ} 02^{\prime}, 7 \mathrm{E} ; 2910 \mathrm{~m}$; 21.07.1975.

- station no 7, prélèvement no 51 : $76^{\circ} 03^{\prime}, 8 \mathrm{~N}-01^{\circ} 27^{\prime}$, 8W; $3713 \mathrm{~m}$; 04.08.1975.

Holotype : 1 우 juvénile provenant de la cavité générale de l'Eurythenes gryllus no $214 \mathrm{BB}$, station no 3 , prélèvement n० 17 .

Les spécimens sont déposés au Muséum national d'Histoire naturelle sous les $n^{0} 176 \mathrm{BB}, 189 \mathrm{BB}$ à $194 \mathrm{BB}, 197 \mathrm{BB}$ à $210 \mathrm{BB}, 213 \mathrm{BB}$ à $215 \mathrm{BB}$.

En raison du mode de fixation (dans le corps de l'Amphipode non ouvert), les Nématodes sont très fragiles et se cassent en plusieurs morceaux au moment de leur extraction; l'holotype a de plus été en partie disséqué pour localiser l'emplacement de la vulve. 
Nématodes de grande taille : longueur variant entre $2 \mathrm{~cm}$ et $16 \mathrm{~cm}$. Chez la plupart des spécimens, la cuticule est largement décollée du corps par suite de la fixation.

Corps robuste, légèrement aminci aux extrémités ; extrémité antérieure arrondie; court stylet saillant légèrement hors de l'ouverture buccale visible chez la plupart des spécimens ( $f i g .2, A, B, C$ ); queue arrondie, munie d'un petit mucron
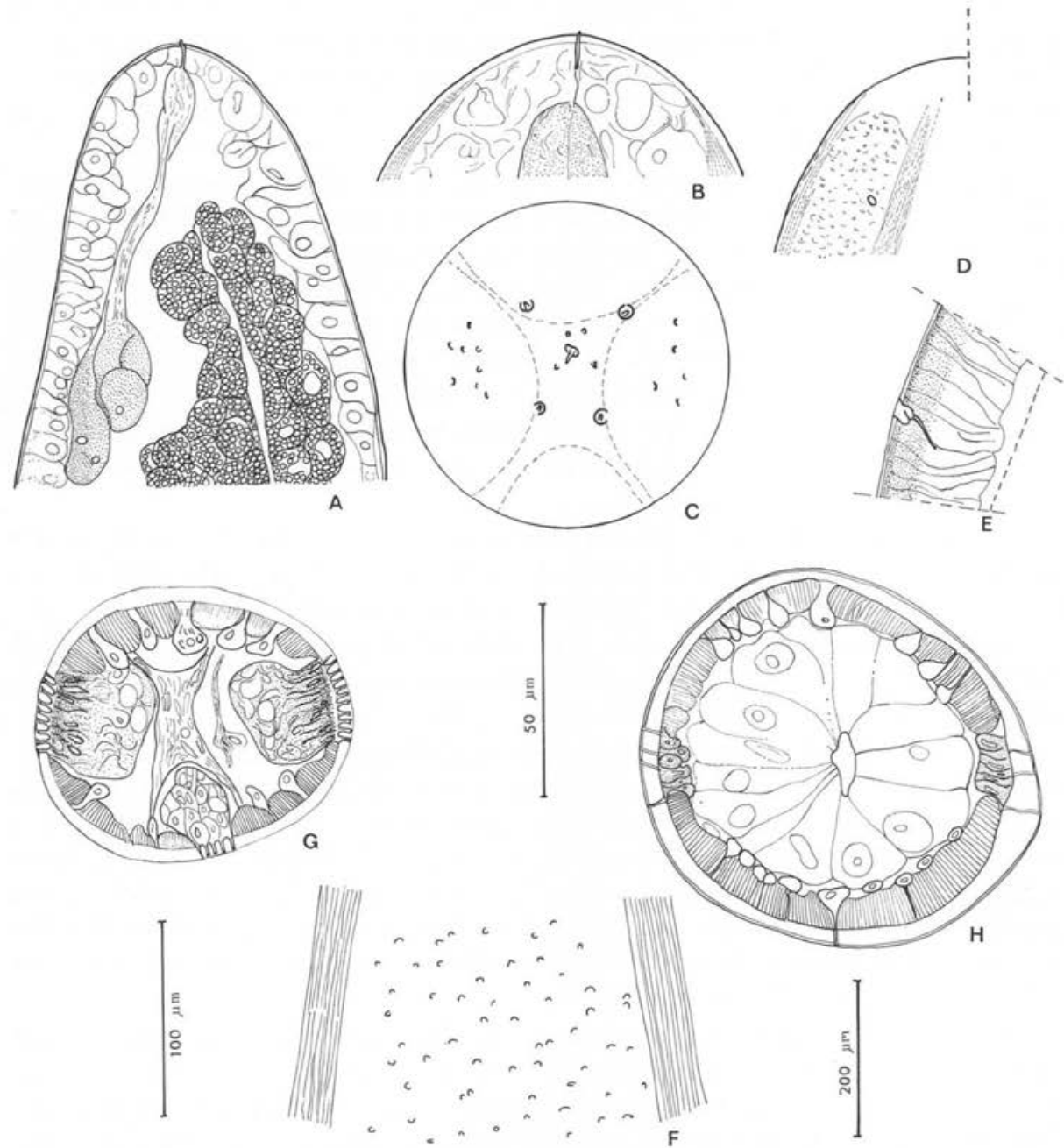

FIG. 2. - A : extrémité antérieure d'un petit spécimen (long de $35 \mathrm{~mm}$ ). B : extrémité antérieure d'un grand spécimen montrant le stylet. C : vue apicale. D : extrémité antérieure, vue superficielle d'un champ latéral montrant l'ouverture amphidiale. E : amphide. F : vue externe de la cuticule au niveau d'un champ latéral. G : coupe transversale au niveau du cordon œsophagien. H : coupe transversale au niveau du trophosome (A, C, E, F : éch. roo $\mu \mathrm{m}$; B : éch. $50 \mu \mathrm{m} ; \mathrm{D}, \mathrm{G}, \mathrm{H}$ : éch. $200 \mu \mathrm{m})$. 
plus ou moins nettement lobé à l'extrémité, dépourvu d'axe cytoplasmique ( $f i g .3, A$ ) : chez le plus petit spécimen, long de $2,2 \mathrm{~cm}$, l'extrémité postérieure est conique, à pointe bifide (fig. 3, B).

Soies céphaliques très petites et fines, visibles seulement en vue apicale, comprenant un cycle interne et un cycle externe de 4 soies submédianes chacun, disposées dissymétriquement par rapport au plan latéral ( $f$ i. 2, C).

Amphides très difficiles à repérer en raison du décollement de la cuticule chez la plupart des spécimens; nous avons cependant pu les observer chez un spécimen : l'ouverture amphidiale, située à $200 \mu \mathrm{m}$ de l'extrémité antérieure, est petite et arrondie ( fig. 2, D); l'amphide est en forme de poche à fond en cul de bouteille, d'où part un fin canal à paroi sclérotisée ( $f i g .2, E$ ).

Cordes hypodermiques latérales larges, avec de nombreuses cellules hypodermiques glandulaires débouchant à l'extérieur par une multitude de petits pores qui criblent la cuticule ( $f i g .2, D, F$ ) ; quelques rangées de pores s'observent également le long de la corde ventrale, qui est bien développée dans la région antérieure et s'amincit ensuite; corde dorsale étroite; entre les cordes latérales et médianes, chacun des 4 champs musculaires submédians est interrompu par quelques rangées de cellules non musculaires, dont le nombre est variable suivant les niveaux (fig. 2, G, $H$ ).

Esophage réduit à un cordon cytoplasmique central allongé dorso-ventralement en section transversale ( $f i g .2, G$ ), terminé par une masse glandulaire visible surtout chez les petits spécimens ( fig. 2, $A$ ).

Trophosome débutant très près de l'extrémité antérieure, surtout chez les grands spécimens, constitué de grandes cellules disposées autour d'une petite lumière centrale; on compte une dizaine de cellules dans une section transversale (fig. 2, H).

Anus réduit à un petit pore arrondi, débouchant dans une vésicule rectale à paroi épithéliale ( $f i g .3, A, D$ ) ; le trophosome est creusé ventralement à ce niveau d'une dépression où se loge la vésicule rectale ( $f i g .3, B$ ).

Appareil génital présentant la disposition habituelle aux femelles de Benthimermithidae : vulve non saillante, située aux environs du milieu du corps; ouverture vulvaire ronde ( $f g . .3, G$ ), court vagin dirigé perpendiculairement à la paroi du corps ; utérus divergents recourbés en crosse à leur extrémité et aboutissant à de longs ovaires récurrents. Chez les grands spécimens, les extrémités proximales des ovaires (extrémités en relation avec les utérus) sont situées relativement près des extrémités du corps ; les ovaires sont très amincis à leurs extrémités aveugles, qui sont situées au voisinage de la vulve ( $f$ ig. $3, C$ ).

Les utérus sont munis dans leur tiers voisin de la vulve de glandes annexes qui sont échelonnées le long de leurs faces latérales (fig. 4, $A, B$ ); chez un grand spécimen, chaque utérus en possède une cinquantaine; chaque glande est constituée de plusieurs grandes cellules à gros noyau glandulaire, et placée au sommet d'un court canal qui débouche dans l'utérus; les glandes sont de taille variable, et peuvent être simples ou plurilobées (fig. 3, E, F) ; les plus grandes atteignent $500 \mu \mathrm{m}$ dans leur plus grand diamètre. La vulve est entourée par de nombreuses petites glandes simples (fig. 3, G). 

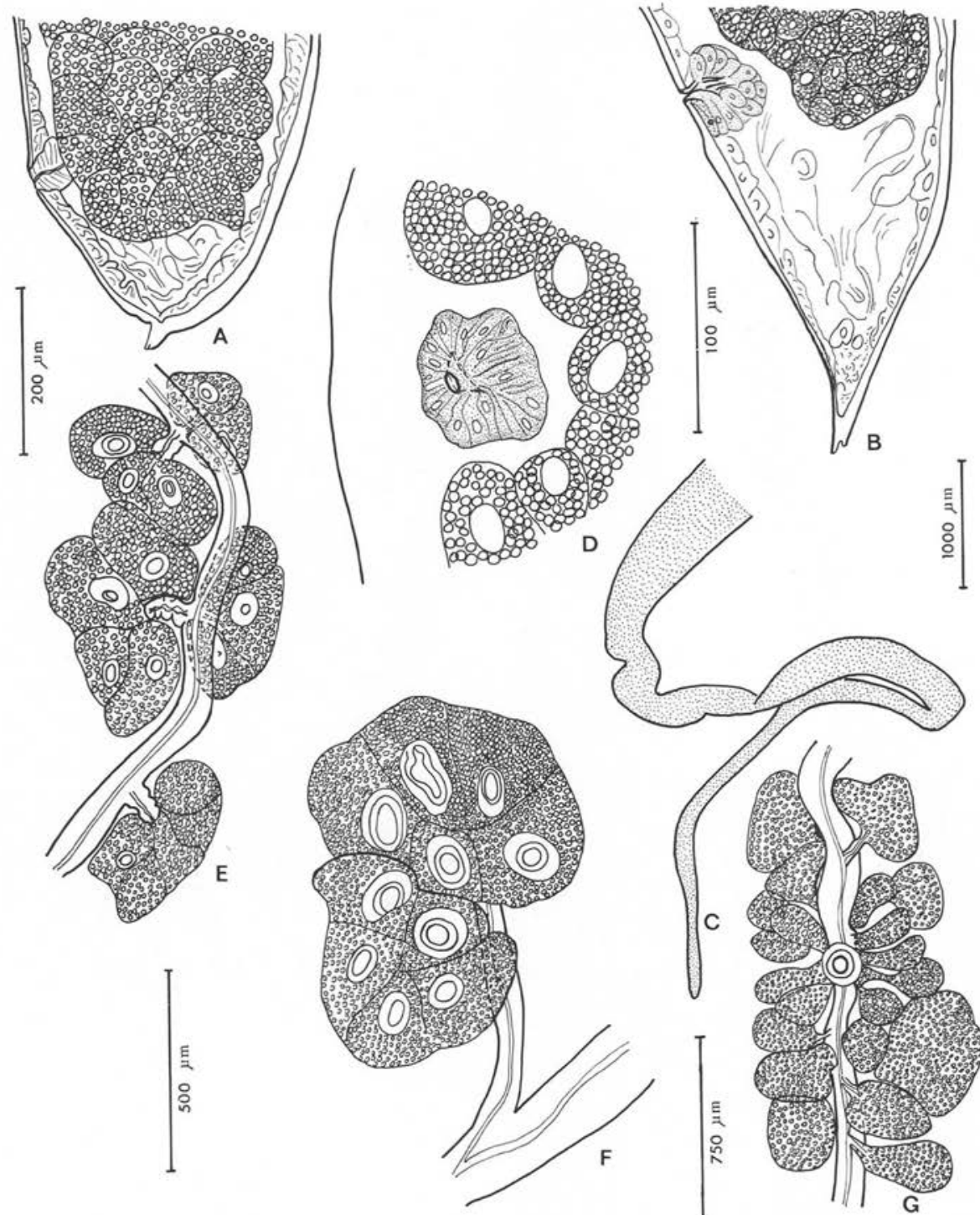

FIG. 3. - A : extrémité postérieure d'un grand spécimen, vue latérale. B : extrémité postérieure d'un petit spécimen long de $2,2 \mathrm{~cm}$, vue latérale. C : extrémité aveugle d'un ovaire. D : anus et vésicule rectale, vue ventrale. $\mathrm{E}, \mathrm{F}$ : portions d'utérus avec glandes annexes. $\mathrm{G}$ : région vulvaire, vue ventrale. (A, F : éch. $200 \mu \mathrm{m} ; \mathrm{B}, \mathrm{D}$ : éch. 10o $\mu \mathrm{m} ; \mathrm{C}$ : éch. $1000 \mu \mathrm{m} \cdot \mathrm{E}$ : éch. $500 \mu \mathrm{m} ; \mathrm{G}$ : éch. $750 \mu \mathrm{m})$. 

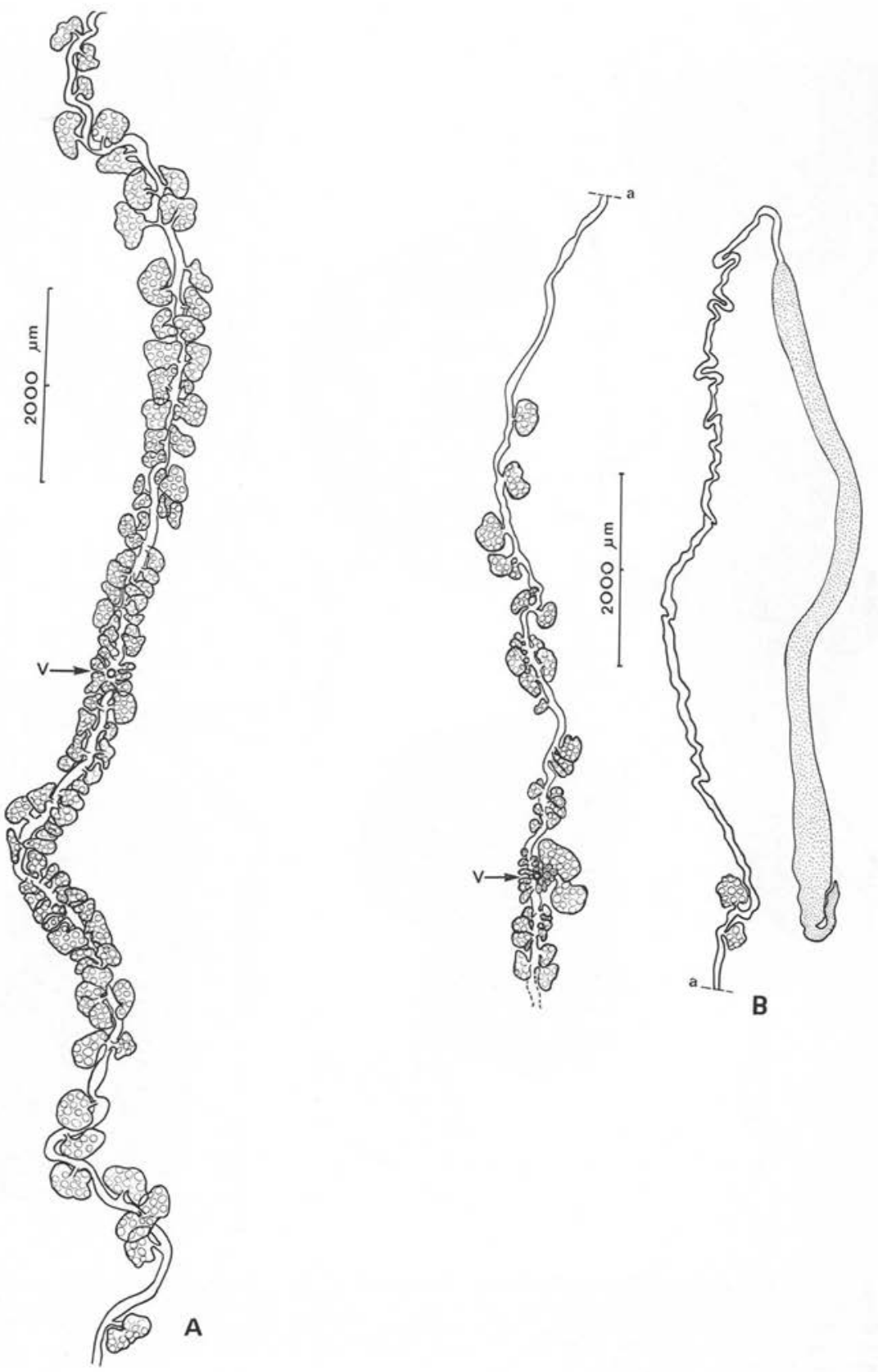

Fig. 4. - A : vulve et parties des utérus antérieur et postérieur munies de glandes annexes chez un grand spécimen. B : ovaire et utérus antérieur d'un petit spécimen. V: vulve. 
Principales dimensions de l'holotype : longueur : $11 \mathrm{~cm}$; largeur au milieu du corps ; $1,3 \mathrm{~mm}$; distance à l'extrémité antérieure : des amphides : $160 \mu \mathrm{m}$; de la vulve : $4,6 \mathrm{~cm}$; queue : $110 \mu \mathrm{m}$ avec un mucron de $20 \mu \mathrm{m}$; distance de l'extrémité proximale de l'ovaire antérieur à l'extrémité antérieure : $5 \mathrm{~mm}$; distance de l'extrémité proximale de l'ovaire postérieur à l'extrémité postérieure : $8,6 \mathrm{~mm}$.

\section{Discussion}

Ces femelles possèdent les principaux caractères de la famille des Benthimermithidae Petter, 1980 : œsophage atrophié, intestin transformé en trophosome, ouverture amphidiales en forme de pore, anus et rectum vestigiaux, vulve médiane, appareil génital femelle amphidelphe avec ovaires récurrents, et au point de vue biologique femelles juvéniles parasites d'Invertébrés marins.

La famille des Benthimermithidae comprend actuellement 2 genres : le genre Abos Rubtsov, 1980 représenté par une espèce dont les femelles sont seules connues, et le genre Benthimermis représenté par 14 espèces dont 5 sont connues uniquement par les femelles (Petter, 1980 et 1982c), 8 connues uniquement par les mâles (Petter 1981a et b, et 1982a, b, c) et une connue par les 2 sexes (Petter, 1982c).

Les femelles décrites ci-dessus diffèrent de toutes les espèces de la famille dont les femelles sont connues par la présence de glandes annexes aux utérus.

Elles s'écartent également de toutes les espèces de la famille par leur grande taille (celle-ci varie de $2 \mathrm{~cm}$ à $16 \mathrm{~cm}$, alors que les plus grandes espèces connues jusqu'à présent ne dépassaient pas $15 \mathrm{~mm}$ ), et par la présence d'un court stylet buccal visible chez certains spécimens; de plus, chez les 2 espèces du genre Benthimermis où le cycle interne de soies céphaliques a été observé (femelle juvénile parasite d'Isopode no 1003 BA, Petter 1980 et mâle de Benthimermis rotundicauda Petter 1981b), ces soies sont au nombre de 6 alors qu'elles ne sont que 4 chez les femelles décrites ci-dessus.

Ces femelles appartiennent donc à une nouvelle espèce ; la présence de glandes annexes aux utérus nous paraît justifier pour cette espèce la création d'un nouveau genre, que nous nommons Adenodelphis n. g. avec pour espèce-type et unique espèce: Adenodelphis eurythenes, n. sp.

Nous modifions la diagnose de la famille des Benthimermithidae telle qu'elle a été donnée par Petter, 1980 en fonction de ce nouveau genre et du genre $A$ bos Rubtsov, 1980, ainsi que des descriptions de mâles du genre Benthimermis faites en 1981 et 1982 :

Benthimermithidae Petter, 1980; Mermithoidea sensu Andrassy, 1974. 4 soies céphaliques submédianes. Ouvertures amphidiales en forme de pore, amphides tubulaires ou en forme de poche. Cordes hypodermiques au nombre de 4,8 ou plus. Cordes latérales et médianes munies de grosses cellules hypodermiques glandulaires. Esophage atrophié. Intestin transformé en trophosome. Anus et rectum vestigiaux ou 
complètement absents. Vulve à peu près médiane ; appareil génital femelle amphidelphe. Appareil génital mâle avec 2 testicules ; 2 spicules égaux ; une rangée ventrale de papilles précloacales.

Femelles juvéniles parasites d'Invertébrés marins, femelles et mâles adultes libres dans les sédiments marins.

Genre-type : Benthimermis Petter, 1980

Autres genres: Abos Rubtsov, 1980

Adenodelphis n. g.

Adenodelphis n. g. : Benthimermithidae. Extrémité apicale munie d'un cycle interne et d'un cycle externe de 4 petites soies submédianes chacun. Court stylet présent. Champs musculaires interrompus par plusieurs rangées de cellules entre les cordes hypodermiques latérales et médianes. Anus et rectum vestigiaux. Ovaires récurrents. Utérus munis de glandes annexes.

Femelles juvéniles parasites d'Amphipodes.

Mâles inconnus.

Espèce-type et unique espèce : Adenodelphis eurythenes n. sp.

Des glandes annexes à l'appareil génital femelle ont été signalées dans quelques cas chez les Nématodes, mais il s'agit toujours de glandes situées au voisinage de la vulve ou du vagin : Goodey (1935) décrit dans le genre Brevibucca une paire de glandes utérines uninucléées situées un peu en avant du vagin; des glandes vaginales ou vulvaires ont également été signalées chez certains Nématodes libres (voir Chitwood et Chitwood, 1950); enfin, de grosses glandes uninucléées entourant la vulve sont présentes chez certaines espèces du genre Benthimermis et chez Abos bathycola.

L'existence de glandes utérines nombreuses et échelonnées tout le long de la partie proximale des utérus telles qu'on les observe dans le genre Adenodelphis n'a à notre connaissance encore jamais été rencontrée chez les Nématodes.

\section{BIBLIOGRAPHIE}

Chrrwood B. G., Chirwood M. B. : An introduction to Nematology. Section I. Anatomy. Revised edit., Monumental Printing Co., Baltimore, Maryland, 1950, 213 p.

GoodEy T. : Brevibucca saprophaga gen. et sp. nov., a nematode from a rotting lily bulb-scale. J. Helminth., 1935, 13, 223-228.

PETTER A. J. : Une nouvelle famille de Nématodes parasites d'Invertébrés marins, les Benthimermithidae. Ann. Parasitol. Hum. Comp., 1980, 55, 209-224.

Petter A. J. : Description des mâles d'une nouvelle espèce de Nématode marin de la famille des Benthimermithidae. Ann. Parasitol. Hum. Comp., 1981a, s6, 285-295.

Petter A. J. : Description des mâles de trois nouvelles espèces de Nématodes de la famille des Benthimermithidae. Bull. Mus. Natl. Hist. Nat., Paris, $4^{\mathrm{e}}$ sér., A, 1981b, 3, 455-465.

Petrer A. J. : Benthimermis gracilis, n. sp., nouveau mâle de la famille des Benthimermithidae (Nematoda). Bull. Mus. Natl. Hist. Nat., Paris, $4^{\mathrm{e}}$ sér., A, 1982a, 4, $71-74$.

Petrer A. J. : Description de deux nouveaux mâles de la famille des Benthimermithidae (Nematoda) de i'Atlantique Sud-oriental. Bull. Mus. Natl. Hist. Nat., Paris, 1982b (sous presse).

PetTER A. J. : Quelques nouvelles espèces du genre Benthimermis (Benthimermithidae, Nematoda) du Sud de l'Océan Indien. Systematic Parasitology, 1982c (sous presse).

RuBTSov I. A. : A new marine parasitic nematode Abos bathycola n. g., n. sp., from priapulids and the position of Marimermithidae in Nematoda. Parazitologiya, 1980, 14, 117-181 (en russe, résumé anglais). 